\title{
Comparative Study between Microscopic and Endoscopic Trans-Sphenoidal Surgery for Growth Hormone-Secreting Pituitary Adenomas with Super-Sellar Extension
}

\author{
Ahmed Sabry Abdelkader Ibrahim ${ }^{1 *}$, Abdel Fattah Abou Alfotoh Shehab ${ }^{2}$, \\ Mohammad Fathy Eissa ${ }^{2}$
}

${ }^{1}$ Neurosurgery Department, Nasr City Hospital of Health Insurance, Cairo, Egypt

${ }^{2}$ Neurosurgery Department, Faculty of Medicine, Al-Azhar University, Cairo, Egypt

Email: *drahsabrynsg84@gmail.com

How to cite this paper: Ibrahim, A.S.A., Shehab, A.F.A.A. and Eissa, M.F. (2020) Comparative Study between Microscopic and Endoscopic Trans-Sphenoidal Surgery for Growth Hormone-Secreting Pituitary Adenomas with Super-Sellar Extension. Open Journal of Modern Neurosurgery, 10, 393402.

https://doi.org/10.4236/ojmn.2020.104042

Received: December 7, 2019

Accepted: August 8, 2020

Published: August 11, 2020

Copyright $\odot 2020$ by author(s) and Scientific Research Publishing Inc. This work is licensed under the Creative Commons Attribution International License (CC BY 4.0).

http://creativecommons.org/licenses/by/4.0/

\begin{abstract}
Background: Advances in the era of modern micro-neurosurgery enabled a reduction of surgical invasiveness and brain retraction which has been defined as minimally invasive or keyhole surgery. Sinonasal endoscopy has brought radical changes in the concepts of pathophysiology and treatment of sinonasal aliments as well as surgical techniques. Aim of the Study: To compare between the use of endoscopic and microscopic trans-sphenoidal approach in resection of growth hormone-secreting pituitary adenomas with Suprasellar Extension. Patients and Methods: This is a prospective study. It had been conducted upon 20 patients having growth hormone secreting pituitary adenoma admitted to Neurosurgery department in Alzar University hospitals and Nasr City Insurance hospital from 2015 to 2018, divided into 2 groups; group A (10 cases) underwent endoscopic endonasal trans-sphenoidal pituitary adenoma resection, while group B (10 cases) operated upon using the standard microscopic trans-sphenoidal pituitary adenoma resection. The inclusion criteria were included: All patients with growth hormone secreting pituitary adenomas showing manifestations of acromegaly, mass effect or hormonal disturbance. Results: This study showed that improvement in outcome was higher in endoscopic group opposed to microscopic group (100\% vs. $71 \%$ improved headache, $80 \%$ vs. $60 \%$ visual improvement, $75 \%$ vs. $20 \%$ fundus improvement and $60 \%$ vs. $30 \%$ field improvement). Conclusion: We concluded that fully endoscopic procedure result in improved rates of complete tumor removal and a reduced incidence of complications, when compared to the microscopic approach.
\end{abstract}




\section{Keywords}

Acromegaly, Pituitary Adenomas, Endoscopic Trans-Sphenoidal Surgery, Microscopic Trans-Sphenoidal Surgery

\section{Introduction}

Acromegaly is most commonly caused by GH-secreting pituitary adenomas. This rare but very serious condition carries at least twice the mortality rate compared with that in the general population [1].

The diagnosis of acromegaly is often delayed. The authors of many older series have reported a delay in the diagnosis of $7-10$ years after the onset of signs and symptoms. Given that tumor size has been established as an important predictor of surgical outcome, early recognition and treatment are considered keys to achieving high rates of remission and avoiding long-term comorbidities [1].

Total surgical removal of GH-secreting tumors offers the possibility of hormonal control of acromegaly as well as amelioration in the associated multisystem morbidities. As such, recent consensus guidelines recommend surgery for the management of acromegaly as the first-line therapy in cases involving tumors likely to be controlled by surgery. Thus, in patients with acromegaly, the goals of surgical treatment are to normalize biochemical markers, eliminate morbidities associated with the disease, relieve mass effect, and subsequently normalize the overall mortality rate [2].

While the microsurgical trans-sphenoidal route has historically been the favored approach to these lesions, the addition of the endoscope to this procedure represents a recent innovation, allowing the surgeon a panoramic view irrespective of the width and depth of the access [2].

Halstead and Hirsch introduced the trans-sphenoidal approach to pituitary lesions in the early 1900s. Cushing perfected and popularized the sublabial transseptal approach shortly thereafter, making modifications that reduced morbidity [3].

With the evolution of endoscopic sinus surgery, many groups have advocated for the use of endoscopy for both trans-sphenoidal exposure of the Sella turcica and resection of pituitary lesions. In the mid 1990s, neurosurgeons began to use rigid endoscopes for resection of pituitary tumors. Since these initial reports, there have been several more case series describing the successful application of the endoscope to the resection of pituitary tumors [3].

The endoscope has several potential advantages compared with traditional techniques. Endoscopy provides additional lighting and a superior field of vision. With the use of angled scopes, one can visualize critical anatomy such as the optic and carotid protuberances as well as the clival indentation, in addition to being able to assess extrasellar extension of pituitary lesions [4].

However, endoscopic resection requires the surgeon to trade the binocular, 
handless microscope for the monocular, more technically challenging endoscope, a technology with which most neurosurgeons are not as familiar. There have been a number of case series directly comparing endoscopic and microscopic surgical results by individual surgeons [5].

We aim in this study to do comparison between the use of endoscopic and microscopic trans-sphenoidal approach in resection of growth hormone-secreting pituitary adenomas with Suprasellar Extension. The points of comparison are many like time of surgery, extension of removal, complications and hospital stay.

\section{Materials and Methods}

This is a prospective study. It had been conducted upon 20 patients having growth hormone secreting pituitary adenoma admitted to Neurosurgery department in Alzar University hospitals and Nasr City Insurance hospital from 2015 to 2018, divided into 2 groups; group A (10 cases) underwent endoscopic endonasal trans-sphenoidal pituitary adenoma resection, while group B (10 cases) operated upon using the standard microscopic trans-sphenoidal pituitary adenoma resection.

\section{Preoperative assessment:}

All of the patients involved in the study have been subjected to preoperative assessment which includes: Clinical assessment thorough: History taking, Systemic examination: To detect picture of endocrinal disturbance in functioning pituitary adenoma and to detect any systemic risk factor, e.g. diabetes milletus, hypertension and cardiological problems. Neurological examination: with special emphasis on cranial nerve examination and cognitive function. Conscious level, Cranial nerves examination, Ophthalmological examination; includes visual acuity, Fundus examination for evidence of papilledema or optic atrophy, and visual field assessment.

The study included 13 males and 7 females, the youngest was 14 years old. This study included most enclosed sellar tumors with supra sellar extension, provided that the tumor extends symmetrically above the sella in the midline. Irregular, multilocular, fungating tumors or eccentric extensions into the frontal, temporal, or posterior fossae were excluded from our study. Both study groups were not similar as regard age, sex, presenting symptoms, previous management, tumor type, shape or extensions, preoperative images or hormonal profile. Selection between both groups (group A \& B) and choice of the surgical approach was in a regular order one case endoscopic and the next is microscopic with inclusion criteria which include: All patients with growth hormone secreting pituitary adenomas showing manifestations of acromegaly, mass effect or hormonal disturbance.

All of the patients involved in the study have been subjected to preoperative assessment which includes: History taking, Systemic, Neurological, Ophthalmological examination and Investigations in the form of Routine investigations, serum Growth hormone or IGF1 if growth hormone is normal and MRI Coronal and sagittal T1 weighted images with and without gadolinium. 


\section{Ethical committee approval:}

The aim and nature of the study were explained for each patient before inclusion. An informed consent was obtained. Then examinations were performed.

\section{Statistical analysis of the data}

Data were analyzed using Statistical Program for Social Science (SPSS) version 18.0. Quantitative data were expressed as mean \pm standard deviation (SD).

\section{Results}

In our study, we collected data and results are showed that there is no statistically significant difference between both studied groups as regard presenting symptoms.

Descriptive analysis of Table 1 reveals that Acromegalic features was the commonest presenting symptom in both endoscopic and microscopic groups; (100\%) respectively followed by visual impairment; $(60 \% \& 80 \%)$ respectively. Although, loss of libido was the most common endocrinopathies; (75\%\& 60\%) respectively.

Table 2 showed that there is statistically significant difference between pre and after 3 months, after 6 months according growth hormone $(\mathrm{ng} / \mathrm{mL})$.

Table 3 showed nonsignificant Relationship between preoperative GH and Postoperative Biochemical remission and Extent of resection of the tumor and method of Management.

Table 4 showed that improvement in out-come was higher in endoscopic group opposed to microscopic group ( $100 \%$ vs. $71 \%$ improved headache, $80 \%$ vs. $60 \%$ visual improvement, $75 \%$ vs. $20 \%$ fundus improvement and $60 \%$ vs. $30 \%$ field improvement).

Figure 1 Post-operative follow-up MRI after 6 months showed that $75 \%$ total removal and $25 \%$ residual removal.

Illustrative cases: (Figure 2 is 1 st MRI images $(a+b)$. Figure 3 is 2 nd MRI images $(a+b))$.

Table 1. Clinical picture of the each study group.

\begin{tabular}{ccc}
\hline & Endoscopic group (A) & Microscopic group (B) \\
\hline Acromegalic features & $10(100 \%)$ & $10(100 \%)$ \\
Visual impairment & $6(60 \%)$ & $8(80 \%)$ \\
Headache & $5(50 \%)$ & $6(60 \%)$ \\
Menstrual irregularity & $3(30 \%)$ & $2(20 \%)$ \\
\hline
\end{tabular}

Table 2. Comparison between pre and postoperative growth hormone.

\begin{tabular}{cccccccc}
\hline $\begin{array}{c}\text { Growth Hormone } \\
(\mathrm{ng} / \mathrm{mL})\end{array}$ & Mean & $\pm \mathrm{SD}$ & \multicolumn{2}{c}{ Paired Difference } & \multicolumn{2}{c}{ Paired sample t-test } \\
\cline { 6 - 8 } & & & Mean & \pm SD & $\mathrm{t}$ & p-value \\
\hline Pre & 29.89 & 18.52 & & & & \\
Early Postoperative & 11.74 & 15.56 & 18.15 & 18.87 & 2.36 & 0.065 \\
After 3 months & 9.95 & 14.30 & 19.94 & 14.45 & 3.22 & 0.005 \\
After 6 months & 8.01 & 9.85 & 21.87 & 11.06 & 4.99 & $<0.001$ \\
\hline
\end{tabular}


Table 3. Relationship between preop. GH and Postop Biochemical remission and Extent of resection of the tumour and method of Management.

\begin{tabular}{|c|c|c|c|c|c|c|}
\hline \multirow[b]{2}{*}{ Pre operative GH } & \multirow{2}{*}{$\begin{array}{c}\text { Post } \\
\text { operative } \\
\text { GH }\end{array}$} & \multirow{2}{*}{$\begin{array}{l}\text { Biochemical } \\
\text { remission }\end{array}$} & \multicolumn{2}{|c|}{ Extent of resection } & \multicolumn{2}{|c|}{ Management } \\
\hline & & & Total & Sub-total & $\begin{array}{l}\text { Microscopic } \\
\text { Trans-nasal }\end{array}$ & $\begin{array}{c}\text { Endoscopic } \\
\text { transnasal }\end{array}$ \\
\hline Total No. (20 cases) & $\leq 1.5 \mathrm{ng} / \mathrm{mL}$ & $8(+\mathrm{ve})$ & 6 & 2 & 3 & 5 \\
\hline$>1.5 \mathrm{ng} / \mathrm{mL}$ & $>1.5 \mathrm{ng} / \mathrm{mL}$ & 12 (-ve) & 9 & 3 & 6 & 6 \\
\hline
\end{tabular}

Table 4. Showing postoperative improvement.

\begin{tabular}{cccc}
\hline Postoperative improvement & Endoscopic, $\mathbf{N}=\mathbf{1 0}$ & Microscopic, $\mathbf{N}=10$ & $\mathbf{P}$ \\
\hline Visual & $8 / 10(80 \%)$ & $6 / 10(60 \%)$ & $<0.05$ \\
Fundus & $3 / 4(75 \%)$ & $1 / 5(20 \%)$ & $<0.01$ \\
Field & $6 / 10(60 \%)$ & $3 / 10(30 \%)$ & $<0.05$ \\
Headache & $10 / 10(100 \%)$ & $5 / 14(71 \%)$ & $<0.05$ \\
\hline
\end{tabular}

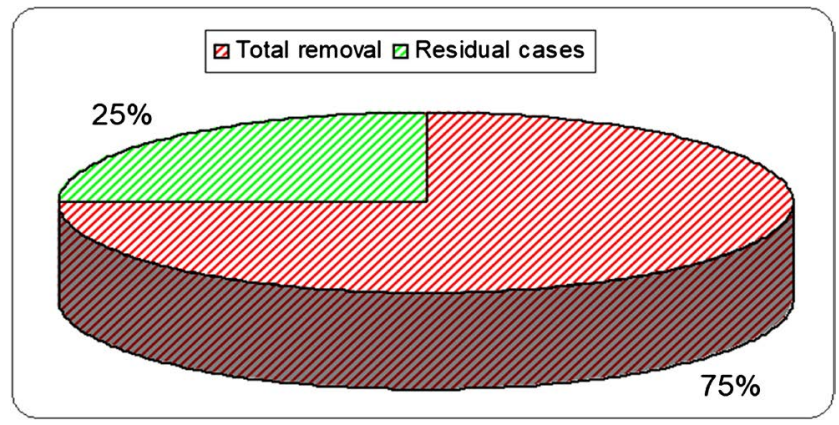

Figure 1. Post-operative follow-up MRI after 6 months.

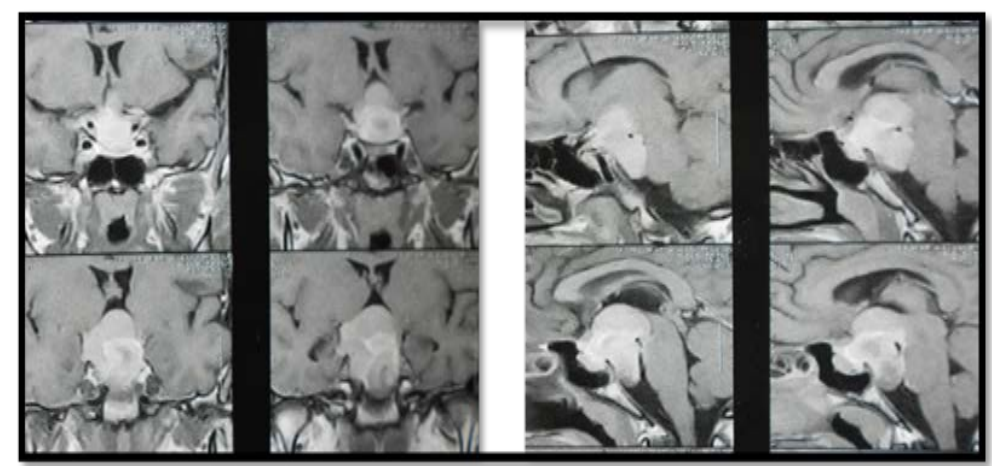

(a)

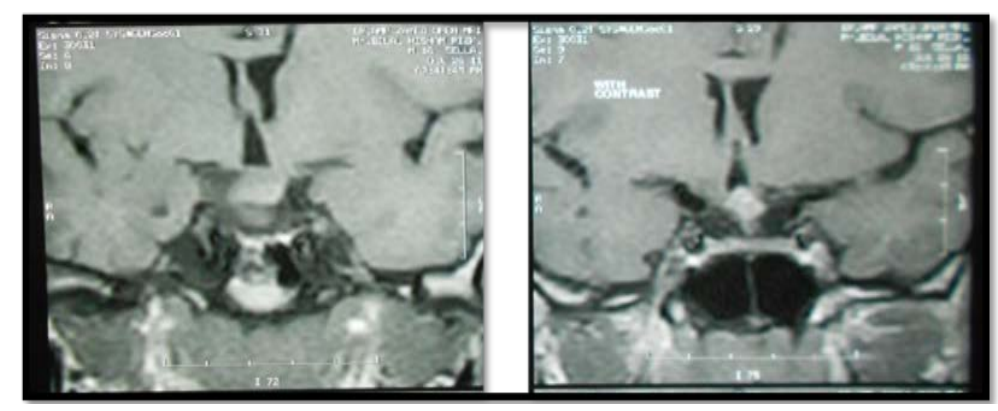




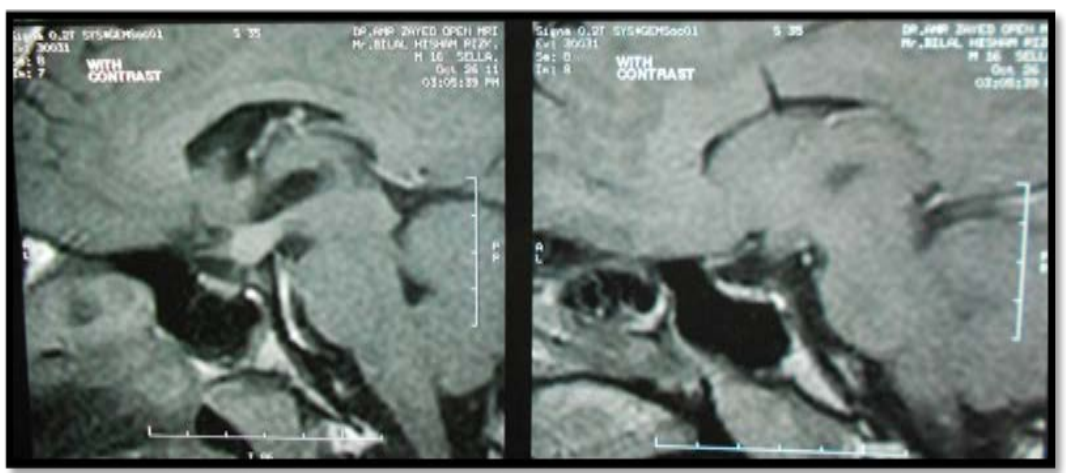

(b)

Figure 2. 18-year-old male presented with acromegalic features, sever headache and visual field defect more in left eye. $\mathrm{GH}$ is $(>40 \mathrm{ng} / \mathrm{ml})$. Patient was operated by microscopic trans septal trans-sphenoidal approach. Post operatively patient showed improvement in headache and visual field. GH is $(15.5 \mathrm{ng} / \mathrm{ml})$. (a) showed preoperative MRI with contrast brain showing pituitary macroadenoma with homogenous enhancement with suprasellar extension and optic chiasm compression while (b) showed postoperative MRI with contrast brain showing total excision of the tumor with free optic chiasm.

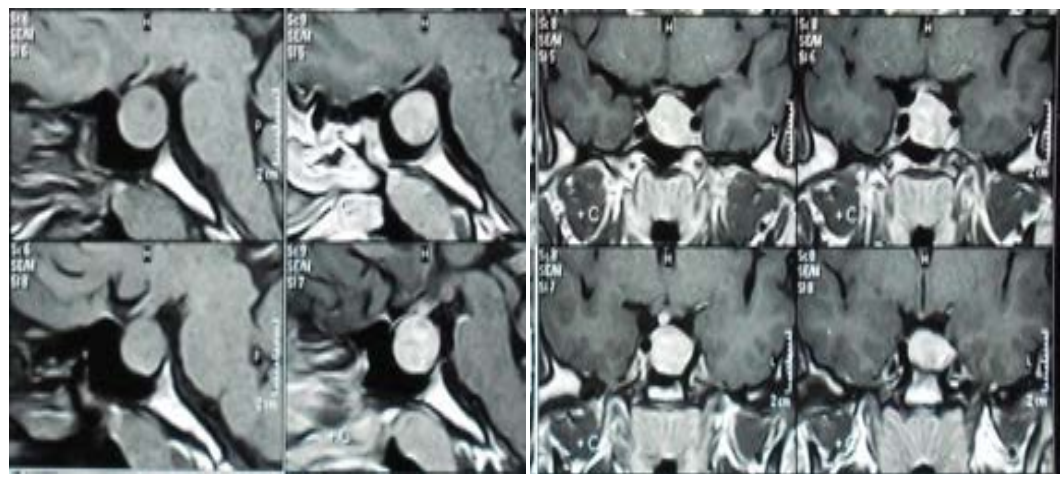

(a)

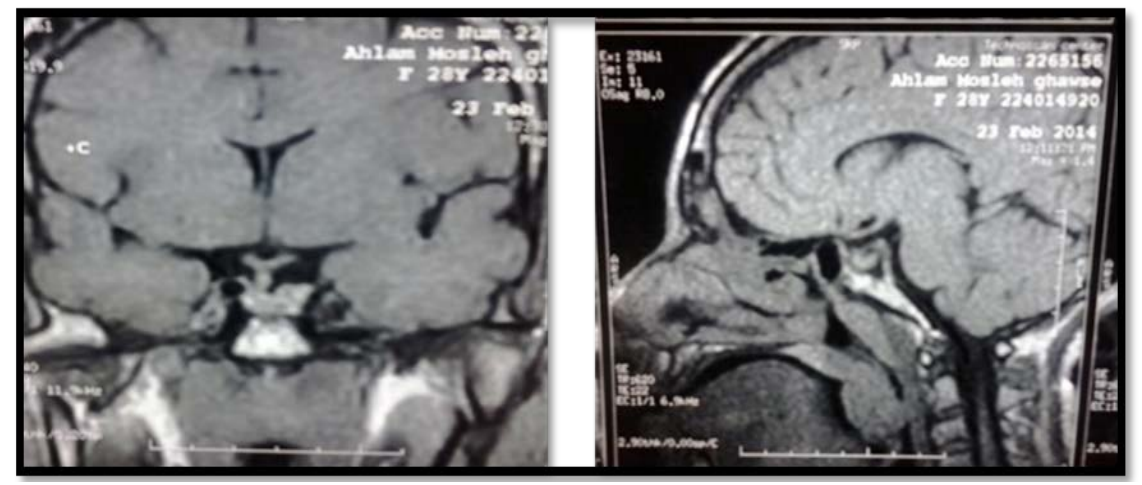

(b)

Figure 3. 30-year-old female presented with acromegalic features, headache and menstrual irregularities. GH is $(19.6 \mathrm{ng} / \mathrm{ml})$. Patient was operated by endoscopic endo nasal trans-sphenoidal approach. Postoperatively patient showed improvement of headache and menstrual irregularities with slight improvement of acromegalic changes. $\mathrm{GH}$ is (4.2 $\mathrm{ng} / \mathrm{ml}$ ). (a) showed MRI with contrast brain showing pituitary macroadenoma with homogenous enhancement while (b) showed MRI brain with contrast showing near total removal of the pituitary tumor with small left lobe focal lesion. 


\section{Discussion}

Therapy for acromegaly is directed at the control of tumor growth, restricting GH hypersecretion, and normalizing IGF-I levels and the long-term objective is to reduce the morbidity and mortality rate to a level comparable to that of the general population. To objectively measure postoperative GH function, typically after an OGTT, IGF-I is measured to assess the biochemical response to treatment [6].

Trans-sphenoidal surgery is an extra arachnoid approach, requires no brain retraction, no external scar (aside from where a fat graft is procured, if used). It is usually the procedure of choice. Indicated for microadenomas, macroadenomas without significant extension laterally beyond the confines of the sella turcica, patients with CSF rhinorrhea, and tumors with extension into sphenoid air sinus [5].

In this study we had 14 cases with multiple sphenoid septae and 6 cases with single sphenoid septa and 16 cases with sellar type sphenoid sinus and only 4 cases with presellar type sphenoid sinus.

Throughout the study, MRI brain with and without contrast was performed before surgery in all patients as the main tool used for identification of the soft tissue relations of the tumor [7]. MRI was also the main tool used throughout the study to judge the extent of surgical radicality being a more solid and reliable evidence.

There was no constant relationship between tumor size and preoperative hormone levels, however, the larger tumors showed a higher level of preoperative prolactin especially in cases with cavernous sinus invasion for prolactin secreting adenomas. Also, there was no relationship between the MRI findings and the pathological nature of the tumor which agrees with the findings of [3].

We performed follow-up MRI 3 months postoperatively, however; some [8] advocates early postoperative MR imaging; as it is useful in the detection of residual tumor. Since the early postoperative sella retains its preoperative volume, one can easily differentiate residual tumor from a normal gland, implanted materials, or postsurgical granulation tissue.

In addition, early postoperative MR images can be an excellent baseline if radiation therapy is necessary for treatment of residual tumor or recurrent tumor suspected on follow-up MR images.

We can summarize the comparison between endoscopic and microscopic approaches in pituitary surgery in the following regarding exposure obtained and surgical experience, we revealed that suprasellar extensions of macroadenomas in conventional microscopic pituitary surgery naturally descent into the sella turcica as the tumors were removed through the sphenoid sinus.

This was facilitated by Valsalva maneuvers conducted by the anesthesiologist, which transiently raise intracranial pressure and cause inferior displacement of the mass. Parasellar extensions of pituitary tumors, however, were more difficult to manage, as the lateral perspectives offered by the microscope were greatly li- 
mited. Given the proximity of the optic nerves, optic chiasm, carotid arteries, and cavernous sinuses to the sella, blind curettage of any tumor remnants extending beyond the limits of the sella wasn't advised and this was similarly advised by [3] [6].

In this study we found 13 cases with homogenous enhancement on post contrast MRI in T1WI, and 7 cases with heterogeneous enhancement, 9 of these 13 homogenous enhanced tumours were totaly resected while 6 of 7 cases with heterogenous enhancement were totally resected,We noticed that the relationship between enhancement of the tumour and the extent of the resection of the tumour is in significant ( $p$-vaule was in significant). We found also that 17 cases with hyper intense adenoma and 3 cases with iso intense adenoma in T2WI MRI.

A healthy nose provides about half of the resistance of the entire respiratory tract. A serious decline in this resistance (resection of the turbinates) might considerably upset the balance of resistance needed for deep pulmonary inspiration and result in shortness of breath that may cause various pulmonary troubles e.g. dyspnea [8].

In our study, improvement in out-come was higher in endoscopic group opposed to microscopic group ( $100 \%$ vs. $71 \%$ improved headache, $82 \%$ vs. $58 \%$ visual improvement and $80 \%$ vs. $55 \%$ total tumor removal as illustrated by follow-up MRI) [2] compared a group of patients who Underwent the sublabial approach with a group who were operated endoscopically and found no differences in out-come regarding vision and anterior pituitary function. Cappabianca [9] compared 10 patients with pituitary adenomas treated endoscopically with 20 patients treated with traditional transseptal trans-sphenoidal surgery and found improved out-come of endoscopic group against microscopic one regarding tumor excision and hormone imbalance. In a series of 46 patients who received a fully endoscopic transsphenoid surgery to the pituitary gland, [10] Jho reported similar results. Preliminary evidence suggests that complication rates and surgical outcomes of our microscopic group were nearly parallel to those that have been reported in large series of transphenoidal microscopic pituitary surgeries.

We finished with a suggestion that, the minimal invasiveness of the endoscopic approach in the presellar phase of the operation, and the safer tumor removal under direct visual control, could offer patients with recurrences the possibility of avoiding or delaying the radiotherapy which often causes secondary hypopituitarism and this is also suggested by Cappabianca and his colleagues [9].

\section{Conclusions}

We found that fully endoscopic procedure may result in improved rates of complete tumor removal and a reduced incidence of complications, when compared to the microscopic approach. We found that the endoscope enabled better diffe- 
rentiation between tumor and normal pituitary tissue than the operating microscope, also better sight of residual cavity after tumor removal and these coincide with what found. They emphasized that the endoscope was helpful for tumor micro-dissection with excellent panoramic vision.

The use of endoscopy in the resection of pituitary tumor has been well described in recent years. More widespread experience with this technique is required before it gains acceptance as preferable to trans-sphenoidal microscopic surgery in the management of pituitary adenomas.

In general, before such a shift in surgical practice can occur several fundamental considerations must be made. First and foremost, there should be some objective evidence that the proposed technique represents an improvement over the old method. In addition, the proposed innovation should involve no undue burden or risk to the patient. Finally, there must be means by which a new surgical technique can be reasonably approximated in a controlled setting for training purposes.

\section{Limitations of the Endoscopy in the Pituitary Surgery}

- The initially reduced ability makes the operative times longer.

- The endoscope provides two-dimensional, flat images, which are inferior to the three-dimensional stereoscopic images provided by the microscope.

- The endoscope offers vision on the video-monitor without a sense of the deepness that can be gained with the surgeon's experience, executing in- and out-movements.

- Dedicated instruments designed for the endoscopic approach are absolutely essential, but not all are readily available, the narrow space for the instruments might create problems in case of complications, such as a profuse hemorrhage or if a suture was required, which is not yet possible.

- The control of the bleeding, either venous or arterial, during the endoscopic procedure may be difficult.

- The introduction of the endoscope and the instruments inside the nostril often causes venous bleeding of the nasal mucosa, especially during the initial attempts, thus requiring continuous irrigation of the lens, which is stressful and time-consuming.

\section{Conflicts of Interest}

The authors declare no conflicts of interest regarding the publication of this paper.

\section{References}

[1] Giustina, A., Barkan, A., Chanson, P., et al. (2010) Pituitary Society; European Neuroendocrine Association. Guidelines for the Treatment of Growth Hormone Excess and Growth Hormone Deficiency in Adults. Journal of Endocrinological Investigation, 31, 820-838. https://doi.org/10.1007/BF03349263

[2] Sheehan, M.T., Atkinson, J.L.D. and Kasperbauer, J.L. (1999) Preliminary Compar- 
ison of the Endoscopic Transnasal vs. the Sublabial Transseptal Approach for Clinically Non-Functioning Pituitary Macroadenomas. Mayo Clinic Proceedings, 74, 661-670. https://doi.org/10.4065/74.7.661

[3] Montanera, W. and Kucharczk, W. (1998) Imaging of Sellar \& Parasellar Lesions in Neurosurgery. In: Wilkin, R.H. and Rengachary, S., Eds., Mc Craw Hill, New York, 1962-1977.

[4] Mortini, P., Losa, M., Barzaghi, R., et al. (2005) Results of Transsphenoidal Surgery in a Large Series of Patients with Pituitary Adenoma. Neurosurgery, 56, 1222-1233. https://doi.org/10.1227/01.NEU.0000159647.64275.9D

[5] Zada, G., Kelly, D.F., Cohan, P., et al. (2003) Endonasal Transsphenoidal Approach for Pituitary Adenomas and Other Sellar Lesions: An Assessment of Efficacy, Safety, and Patients Impressions. Journal of Neurosurgery, 98, 350-358.

https://doi.org/10.3171/jns.2003.98.2.0350

[6] Benveniste, R.J., King, W.A., Walsh, J., et al. (2005) Repeated Transsphenoidal Surgery to Treat Recurrent or Residual Pituitary Adenoma. Journal of Neurosurgery, 102, 1004-1012. https://doi.org/10.3171/jns.2005.102.6.1004

[7] Vieira, J.O., Cukiert, A. and Liberman, B. (2004) Magnetic Resonance Imaging of Cavernous Sinus Invasion by Pituitary Adenoma, Diagnostic Criteria and Surgical Findings. Arquivos de Neuro-Psiquiatria, 62, 37-43. https://doi.org/10.1590/S0004-282X2004000300011

[8] Yoon, P.H., Kim, D.I., Jeon, P., et al. (2001) Pituitary Adenomas: Early Postoperative MR Imaging after Transsphenoidal Resection. American Journal of Neuroradiology, 22, 10-19.

[9] Cappabianca, P., Alfeiri, A., Calao, A., et al. (1999) Endoscopic Endonasal Transsphenoid Approach: An Additional Reason in Support of Surgery in the Management of Pituitary Lesions. Skull Base Surgery, 9, 109-117. https://doi.org/10.1055/s-2008-1058157

[10] Jho, H.D. (1999) Endoscopy in Skull Base Surgery. Proceedings of the 4th European Skull Base Congress, Nuremberg (Germany), May 19-22, 1999. Skull Base Surgery, 9, 20-21. 